\title{
Exploration of the Accessible Chemical Space of Acyclic Alkanes
}

\author{
Robert S. Paton and Jonathan M. Goodman*
}

\section{Supporting Information}

Isomer numbers (all digits)

$\mathrm{C}_{1000} \mathrm{H}_{2002}$ :

73535427719085205624005711400038036158183109301677218945485369141845635640573453701 26384596274247734352048089098710726217236720772089521031575700080791919614143683611 69185118850238645168266986819849039203846909534487733979262106798185165148591369697 59121567206036734308211389421957112003345998339163184077197276837940533504142663855 22285497381742331952500813776473702316906830629777627612978475010672965670707321860 538101657368032050644746140 (7.3535428 E441) possible structural isomers.

72142147369250171601872065936081600258268610962089125646345215406283784248490586879 70110463079148554586198502793953266078561719231508715774620374183686175445778401270 66450844694896223842858613302333385358181582929821922762992769309331818769572033496 57018411607519787402541064698215985933466249577428458798034624262410979275451710272 27796245741033764881923639254657839716739264230693465811769509992547608748334983012 443302087470293280660300821 (7.2142147 E441) allowed structural isomers (C17 filtered).

62074768327378901927480931690692906407473376509861531103614095328560236035955533230 27485850277323386447259450527287007434305296704416604138060383750268284284157960465 30587271171216302003066222617185427528671862769672240358736373267430704908549730966 67109530229237812511630582079708580462625095059028406825768852314767517792586028261 02214143800070161096881273962217731263044072955306514500597339216248158250156552694 419302262095578112961125728 (6.2074768 E441) allowed structural isomers (C16 filtered).

59346473763720381691801218759706507776818493483915461650201500411401814272492448028 99834359752890198101020551069720737831676624904767111098898981764184800575282780232 71865639796911607829966151421675641322754090343091855520557084045196482045994200634 51592537348807583806296108614385564522556079344677973481016125446655940509538247134 20672742140608673964665187085769897909669295672126439531888403663769725900052103182 64476668373770563243537770967641321730338193597794343872962155338683686240308012350 69289139241 (5.9346474 E508) possible stereoisomers.

59043362108000757425687858137948473677314859992148648729418709907551881037010545859 72498866846594684120969850123761015571003232900019720323077945216274108629291383353 49290553491025045033168047435851291341758948315317523430649692741219386212965580114 77688349613181035591790525101869778042957398883948703382511132828580073422479636955 27354383658821760752134284544082437536887426205728633049001093983472967534840826798 79661819453697036528557144638355824968854671371396376415225731498672710568036323877 99034773303 (5.9043362 E508) allowed stereoisomers (C17 filtered).

55432096077750773209922158245247961933621365467020031021146270313114939706997082404 06328579123390039529063605682291806564506705983342737528232316734860785912149569557 70526084054245237724582525565217227067672949918525391491607425713170873600291845479 82412240179040853731332723861574735192003810100560812419866955282444673130238690598 32082248967476251788417405576882885410824516063748855644439241100079667807122929416 07232440962993475147432634658343199848567094317829839044175978715834665821966093873 22522427734 (5.5432096 E508) allowed stereoisomers (C16 filtered).

$\mathrm{C}_{2000} \mathrm{H}_{4002}$ :

46055365780053738198952707698879083863596779298840094190780781111467180240519976657 64632029118630310965786615492977537200030083356568794250344607292819644247068818824 
21666981034969349224199533622070089644457233223224152176118054829241290258392602989 37149736411408295857971010358694026768955916908859227192684260378228556583584492256 79347066870920217123408330712879303321381440983573323575101985567068299276763881623 04302050571820405421820166284290749556972087256171440570212114946523605235375506738 82801601528053964382857856855058061802921101859639626686287667483049421645616916651 36352525340555136914146809149283898000440704107469422426075058402106804810449698363 39392429246949035960847037306894511585670557328078740769225801436712030001756685659 80209626454630437524009863928785903379740203834798673670132617521795135936278520256 5738081172698363317702430905743880911668210777061802350870067 (4.6055366 E890) possible structural isomers.

44284767774456543181962887278668923392447320559917160381735705074792768736950870997 91034780036471238015078837804624629484462753306374846041586495216574144349553124437 37622067607463343676351089075888135200100577169341127029659484017877465824927897298 82012737591831570357031685367521913308353589001240366237028131468782346491652437401 76696304395000374986677921654151252561049339364809824334920637163509241352023211492 40863039312172565588973578646154932412057236253013822153714447897929219890831297863 03809383428978915027292372676348931707749762022284877362662381784501453984557837814 81454023110535320661572331167781505430342841242303766981302406201528635115375449923 78119855415260717548502208189653943134788370231878775862335418454287938414455347766 45557538988652499568596349088226237448883915136743643868210953251615489445459676936 5062169098415170664816628164523840488301092982107923772548743 (4.4284768 E890)

allowed structural isomers (C17 filtered).

32404729951238680391675684772764283941852074535729539069572009975016710843822440821 02141458092155479585702131896625443317523857461909278061237335623246380567257150314 10408999876582975280041553626275016787098748907947111183454988737438077737966924496 10232359943900895845752935069672805184446529375338806161994706150195244219153247717 35364349457163679443292753674774441368617083454176302140355138491040599194519048420 87246123872839415855416019674143235573685475574423689644070203786014132797249874736 74477985067496849488898615621155295759960593202367546290340283128168778433207244807 99504548080835358603700543344913263090674734737079157732759648927319307524415996889 05067605067240753568749010273724496255465269749506737668299074470377694896001893558 64131835350349253304848834027622915009319607619001221102662587415623929204074511769 5399668654090901336104761868239208745284603269866916813735627 (3.240473 E890) allowed structural isomers (C16 filtered).

68465857595045926351858025635445316511124446531480250115741994294545018926474642807 79055801064481848089781019030264013589257942273929024558854936605295003884191940943 11122251154370779687390202375506032182661232719848983936255136382510124724499461639 74245393034984805825463743424529250280446188863359804320231551255163784490658464773 96769700214080650019334955502923780704928913554964792883737739058716424463159674204 78525895329926277620212169201927106047196472518374549286500357523361925684384162968 98769054377956185448406381667065751461140204804440385552560965630765475843945865931 02917838607250027531657635317897719714088083928809055968178735942527486157740465996 36517598650704218723789463219602577836663413285204293434788075750154019072837400017 74756779774484405030629077290911779245283042760238871452528124078242242768439748016 17276993569937665174521535984336337118675187925948102507018044072029449940603820872 29559884805881188956499912171567199690054643410538590436278290967985658031897833096 90516659023426062856497687498 (6.8465858 E1024) possible stereoisomers.

67747386138492979158128927468932423016930419966607489899594366634335322179986076386 24408124652658004277947486209980587037833295431287376195707896136219818038327727847 53357856304679651546246575550747567152271019668989349829883416784616399675926905704 60118331008546867430879521441430228108141193415485602685240733942744007773605807442 27595299757688166290280048299459127095536132057946061681911848771841347433507469010 67955026365736689435430450795318849824873353220694584550048018481665836238761108721 05169241437568314549275244213490043795930313563566125832476343988366835794277920953 95705735053096214438614083313058514476628781618165448761674773827712072271999052237 94901784421053261343343280960661797708774909109526720981328226347655669947265948365 
83692402830401008770888401244060403442340263067338702525030993620430714163241788370 91163580260285295805205154108418313226191334074271988617611890660498350527479893538 02168489566932896359103271459493056657492962273372245226464537432749786219871015755 92920711707152488448374251835 (6.7747386 E1024) allowed stereoisomers (C17 filtered).

59389249697165220699550655303711500493012993511951705214292157221694665272337237803 24468257865636395619639885850329551572242116106986161900765169990702652041395246366 52230357649451154607083596709145750269371131769864167875561795301210297562553353731 45860059414895753059975416824863006493487351068612312470808103393063693538872373522 74478716422978484098172795800430421903159594069586662108602481703075836741194221287 93426816304422399220434286433185644829552368902953316116739114707337293038366850606 26920520765911643044615246219205330876941293781025528021874866810354465424512860061 64761305323698831939749572094297648307602313062517848812089037921935502145791666608 86554822671389936117567179479126792885069483092069765906126547294964585999253394783 49034038505814365783228878607122312902375911742212307285250782725985007425117901262 01755341715065247846435029207914133627606235028827236257297299064103285197235358823 11245629836192948238377372300210864787885905556279208020582339726446047415912893939 00509257458205676730215838843 (5.938925 E1024) allowed stereoisomers (C16 filtered).

Enumeration Algorithm in full for filtering C16 substructure:

$$
\begin{aligned}
& \left.N^{\prime}(n)=\sum_{2 a=n}\left(\begin{array}{c}
T s^{\prime}(a)+1 \\
2
\end{array}\right)+T s^{\prime}(a)\left(T r^{\prime}(a)-T s^{\prime}(a)\right)+T t^{\prime}(a) S^{\prime}(a)\right) \\
& +\sum_{\substack{a<b<c<d \\
a+b+c+d=n-1}}\left(\begin{array}{l}
\left(\operatorname{Tr}^{\prime}(a)-T s^{\prime}(a)\right)\left(T r^{\prime}(b)-T s^{\prime}(b)\right)\left(T r^{\prime}(c)-T s^{\prime}(c)\right)\left(T r^{\prime}(d)-T s^{\prime}(d)\right. \\
+\left(T r^{\prime}(a)-T s^{\prime}(a)\right)\left(T r^{\prime}(b)-T s^{\prime}(b)\right)\left(T r^{\prime}(c)-T s^{\prime}(c)\right) S^{\prime}(d) \\
+\left(T r^{\prime}(a)-T s^{\prime}(a)\right)\left(T r^{\prime}(b)-T s^{\prime}(b)\right) S^{\prime}(c)\left(T r^{\prime}(d)-T s^{\prime}(d)\right) \\
+S^{\prime}(a)\left(T r^{\prime}(b)-T s^{\prime}(b)\right)\left(T r^{\prime}(c)-T s^{\prime}(c)\right)\left(T r^{\prime}(d)-T s^{\prime}(d)\right)
\end{array}\right) \\
& +\sum_{\substack{b<c<d \\
b+c+d=n-2}}\left(R^{\prime}(b) R^{\prime}(c) R^{\prime}(d)-\left(R^{\prime}(b)-T s^{\prime}(b)\right)\left(R^{\prime}(c)-T s^{\prime}(c)\right)\left(R^{\prime}(d)-T s^{\prime}(d)\right)\right) \\
& +\sum_{\substack{b<c<d \\
b+c+d=n-1}}\left(R^{\prime}(b) R^{\prime}(c) R^{\prime}(d)-\left(R^{\prime}(b)-T t^{\prime}(b)\right)\left(R^{\prime}(c)-T t^{\prime}(c)\right)\left(R^{\prime}(d)-T t^{\prime}(d)\right)\right)
\end{aligned}
$$

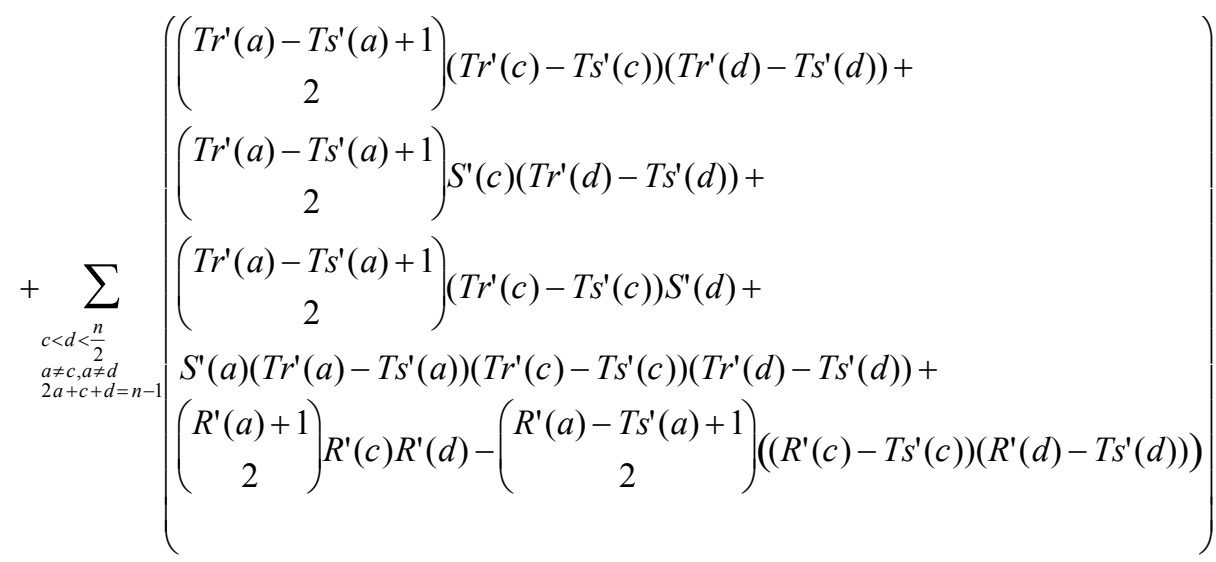$$
+\sum_{\substack{d<\frac{n}{2} \\
a \neq d \\
2 a+d=n-1}}\left(\left(\begin{array}{c}
R^{\prime}(a)+1 \\
2
\end{array}\right) R^{\prime}(d)-\left(\begin{array}{c}
R^{\prime}(a)-T t^{\prime}(a)+1 \\
2
\end{array}\right)\left(R^{\prime}(d)-T t^{\prime}(d)\right)\right)
$$ 


$$
\begin{aligned}
& +\sum_{\substack{a<c \\
a \neq c \\
2 a+2 c=n-1}}\left(\begin{array}{c}
\left.\left(\begin{array}{c}
\operatorname{Tr}^{\prime}(a)-T s^{\prime}(a)+1 \\
2
\end{array}\right)+\left(\begin{array}{c}
\operatorname{Tr}^{\prime}(a)-T s^{\prime}(a)+1 \\
2
\end{array}\right)\left(T r^{\prime}(c)-T s^{\prime}(c)\right) S^{\prime}(c)+\right) \\
2
\end{array}\right) \\
& +\sum_{\substack{a<c \\
2 a+2 c=n-1}}\left(\left(\begin{array}{c}
R^{\prime}(a)+1 \\
2
\end{array}\right)\left(\begin{array}{c}
R^{\prime}(c)+1 \\
2
\end{array}\right)-\left(\begin{array}{c}
R^{\prime}(a)-T s^{\prime}(a)+1 \\
2
\end{array}\right)\left(\begin{array}{c}
R^{\prime}(c)-T s^{\prime}(c)+1 \\
2
\end{array}\right)\right) \\
& +\sum_{a \neq d<\frac{n}{2}}\left(\begin{array}{l}
\left.\left(\begin{array}{c}
\operatorname{Tr}^{\prime}(a)-T s^{\prime}(a)+2 \\
3
\end{array}\right)\left(\operatorname{Tr}^{\prime}(d)-T s^{\prime}(d)\right)+\left(\begin{array}{c}
\operatorname{Tr}^{\prime}(a)-T s^{\prime}(a)+2 \\
3
\end{array}\right) S^{\prime}(d)+\right) \\
\left(\begin{array}{c}
\operatorname{rr}^{\prime}(a)-T s^{\prime}(a)+1 \\
2
\end{array}\right) S^{\prime}(a)\left(\operatorname{Tr}^{\prime}(d)-T s^{\prime}(d)\right)
\end{array}\right) \\
& +\sum_{a \neq d<\frac{n}{2}}\left(\left(\begin{array}{c}
R^{\prime}(a)+2 \\
3
\end{array}\right) R^{\prime}(d)-\left(\begin{array}{c}
R^{\prime}(a)-T s^{\prime}(a)+2 \\
3
\end{array}\right)\left(R^{\prime}(d)-T s^{\prime}(d)\right)\right) \\
& +\sum_{\substack{a=1 \\
3 d=n-1}}\left(\left(\begin{array}{c}
R^{\prime}(d)+2 \\
3
\end{array}\right)-\left(\begin{array}{c}
R^{\prime}(d)-T t^{\prime}(d)+2 \\
3
\end{array}\right)\right) \\
& +\sum_{4 a=n-1}\left(\begin{array}{c}
\left(\begin{array}{c}
\operatorname{rr}^{\prime}(a)-T s^{\prime}(a)+3 \\
4
\end{array}\right)+\left(\begin{array}{c}
\operatorname{Tr}^{\prime}(a)-T s^{\prime}(a)+2 \\
3
\end{array}\right) S^{\prime}(a)+ \\
\left(\begin{array}{c}
R^{\prime}(a)+3 \\
4
\end{array}\right)-\left(\begin{array}{c}
R^{\prime}(a)-T s^{\prime}(a)+3 \\
4
\end{array}\right)
\end{array}\right)
\end{aligned}
$$

Where the terms are the same as those defined in the main text. As in the case of filtering C17, subtracting $N^{\prime}(n)$ from the number of isomers with all possible centroids (similarly enumerated using only allowed rooted trees $R^{\prime}(n)$ ) then yields the number of structural isomers which do not contain the $\mathrm{C} 16$ substructure anywhere in the molecule. 